\title{
Behavioral Considerations In Financial Decisions: Betting Against The Spread In Sports
}

William B. Joyce, (E-mail: wbjoyce@eiu.edu), Eastern Illinois University

\begin{abstract}
People often choose intuitive rather than equally valid non-intuitive alternatives. This research suggests that these intuitive biases arise because intuitions often spring to mind with subjective ease, and the subjective ease leads people to hold their intuitions with high confidence. An investigation of predictions against point spreads found that people predicted intuitive options (favorites) more often than equally valid (or even more valid) non-intuitive alternatives (underdogs). Critically, though, this effect was largely determined by people's confidence in their intuitions (intuitive confidence). Across a naturalistic sample, it is found that decreasing intuitive confidence reduced or eliminated intuitive biases. This finding indicates that intuitive biases are not inevitable but rather predictably determined by a contextual variable that affects intuitive confidence.
\end{abstract}

\section{INTRODUCTION}

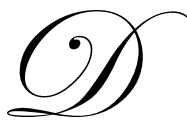

ecisions often require the integration of an intuition (the first answer that springs to mind when one is required to make a decision) with information that invalidates or opposes that intuition. For example, an experimental participant who watches a fellow student give a political speech must integrate the knowledge that the speaker was assigned to give that speech in order to decide the speaker's true attitude toward the speech topic (e.g., Jones and Hams, 1967). A citizen with a favorable intuition about a competent-looking political candidate must integrate negative information about the candidate (e.g., the candidate's views on foreign policy) in order to make a voting decision (e.g., Todorov et al., 2005). And a manager who favorably judges a job candidate during a short interview must integrate other unfavorable information about the candidate (e.g., knowledge that the candidate was fired from his or her last job) in order to make a hiring decision.

In this article, research is presented that sheds light on how people make decisions such as these. How do people decide between intuitive and non-intuitive alternatives when they are aware of information that invalidates or opposes their intuitions?

\section{INTUITIVE VERSUS NON-INTUITIVE DECISION MAKING}

People often choose in line with their intuitions even when other information undermines their intuition's validity (e.g., Denes-Raj and Epstein 1994). This tendency is at the root of a variety of psychological phenomena, including biases in likelihood judgments (Kahneman and Frederick, 2002), the correspondence bias (Gilbert, 2002), the hindsight bias (Fischhoff, 1975), the spotlight effect (Gilovich, Medvec, and Savitsky, 2000), the illusion of transparency (Gilovich, Savitsky, and Medvec, 1998), the above-average and below-average effects (Kruger, 1999), belief in explicitly false statements (Gilbert, 1991), the solo comparison effect (Moore and Kim, 2003), the ratio-bias phenomenon (Denes-Raj, Epstein, and Cole, 1995), perspective-taking failures (Epley et al., 2004), the use of clinical versus actuarial prediction (e.g., Dawes, Faust, and Meehl, 1989), the popularity of sport utility vehicles (Gladwell, 2004), and more (for a review, see Kahneman, 2003). 
Researchers believe that these intuitive biases arise from the interaction of two mental systems: termed System One and System Two (Kahneman, 2003). System One is a relatively effortless system that relies on prior knowledge, judgmental heuristics, immediate experience, and affect in order to rapidly and crudely assess the decision alternatives. This assessment is often accomplished by answering an easy question (e.g., How did the candidate perform during the interview?) where a difficult one is required (e.g., How will the candidate perform the job?; Kahneman and Frederick, 2002). The purpose of this intuitive assessment is to quickly suggest which option should be chosen.

System Two is a slower, effortful, resource-dependent, rule-based system that monitors and updates System One's assessment in light of information that System One neglected to consider. When information opposes System One's assessment, System Two is called on to correct that assessment in an effort to improve decision accuracy. Extant theorizing indicates that despite System Two's corrective attempts, final decisions are often biased by initial intuitive assessments, even when decision makers are aware of information that undermines the validity of those assessments. Thus, in the competition between System One and System Two, System One wins more often than not (e.g., Kahneman, 2003).

Why does System One intuition often prevail over System Two reasoning? The answer depends on the theory. According to some dual-process theories, including the heuristic systematic model (HSM; Chen and Chaiken, 1999) and the elaboration likelihood model (ELM; Petty and Wegener, 1999), invalid System One responses pervade judgment and choice because people are often unmotivated (i.e., cognitively lazy; e.g., Cacioppo and Petty, 1982) or unable (i.e., cognitively overloaded; e.g., Gilbert, Pelham, and Krull, 1988) to fully process relevant information in the judgment context. Without either the motivation or ability to process information that opposes the intuition, decision makers must rely on their initial intuitive, or heuristic, evaluations of the decision options. System One biases emerge from a cognitive busyness or laziness that fails to fully engage System Two, and the fact that decision makers are often cognitively lazy or busy accounts for the ubiquity of intuitive biases.

Even when neither laziness nor busyness are at stake, a different brand of dual-process model (anchoring and adjustment) holds that System One biases emerge from intrinsic insufficiencies in System Two corrections. According to this theory, perceivers start with an intuition (the anchor), and then they serially adjust for information that suggests that the intuition might be invalid. Because perceivers settle on the first answer that seems plausible, their adjustments are insufficient, and they wind up choosing in line with their intuitions (e.g., Epley and Gilovich, 2006). According to this model, intuitive biases arise not only when people are too lazy or busy to use System Two to make corrections. Rather, System Two lacks the capacity to correct enough.

Both of these perspectives offer empirically powerful and highly compelling explanations for why a potentially invalid intuition might win out to influence judgment and choice. It is important to note, however, that these explanations do not, either in isolation or in combination, provide a satisfying account for some varieties of intuitive biases. First, although the HSM and ELM can easily explain why cognitively lazy or busy decision makers often rely on their intuitions, they cannot explain why cognitively motivated and able decision makers often rely on their intuitions as well (e.g., Pelham and Neter, 1995). For all of their strengths in predicting when perceivers will process relevant information, the HSM and ELM fail to predict the perseverance of intuitive biases when all relevant information has been processed.

Second, the distinction between judgment and choice limits the application of anchoring and adjustment models. These models provide a compelling account of how people make continuous judgments (e.g., frequency and percentage estimates; Epley and Gilovich, 2006), but the contention that people serially adjust for counterintuitive information along a continuous dimension means that these models offer a poor account of choices (Epley et al., 2004). For people to serially adjust judgments along a continuous dimension, there must first be a continuous dimension along which adjustment can take place. Thus, to apply anchoring and adjustment theories to dichotomous choice tasks, one must assume that decision makers spontaneously convert choice tasks into judgments along a continuous scale: (see Epley et al., 2004, p. 337). Contrary to this assumption, however, people 
treat choice and judgment tasks quite differently, as they rely on distinct mental processes to handle them (e.g., Simonson and Tversky, 1992). Indeed, the parsimonious hypothesis that people treat choices as choices rather than as continuous judgments has not been disconfirmed. As a result, anchoring and adjustment models cannot easily account for choice phenomena.

An additional limitation of anchoring and adjustment theories is their inability to predict when intuitive biases will emerge and when they will not. One version of anchoring and adjustment holds that intuitive biases are inevitable because adjustments are always insufficient, whereas a weaker version holds that the biases are nearly guaranteed because adjustments are mostly insufficient (Epley and Gilovich, 2004). Neither version offers a general account that predicts when adjustments will be sufficient. Indeed, the mechanisms that are posited to account for sufficiency in adjustments tend to be idiosyncratically linked to particular items and to define sufficiency with respect to accuracy rather than, as we do, with respect to the intuition or anchor.

In sum, intuitive biases exist even when people fully process information suggesting that their intuitions are invalid and even when they are making choices. Neither the HSM, ELM, or anchoring and adjustment model can explain why this is so. In this article, we propose a unique dual-process model of choice that addresses both of these issues. In so doing, we forward a theory that not only explains why intuitive biases arise in motivated choice contexts, but also makes novel predictions about when they will fail to arise. Thus, contrary to anchoring and adjustment theories, our theory suggests that intuitive biases are predictably non-universal: They arise because people feel very confident in their intuitions, so that seemingly robust intuitive biases will fail to arise when confidence in the intuition is undermined.

\section{THE ROLE OF INTUITIVE CONFIDENCE}

This description of how people choose between intuitive and non-intuitive alternatives begins with standard dual-process assumptions. It is assumed that people conduct a cursory assessment of the decision options, and we refer to the output of this assessment as an intuition. It is further assumed that this intuition serves as a decision default (Epstein, 1994) and that the decision default is chosen when people are either unmotivated or unable to engage in further processing (e.g., Chaiken et al., 1989). These basic assumptions are consistent with a wide variety of dualprocess models (e.g., Gilovich et al., 2002) and are relatively uncontroversial.

This account is designed to explain how motivated and able decision makers integrate their intuitions with information that opposes their intuitions in order to arrive at a decision. It is suggested here that because intuitions serve as decision defaults, decision makers' primary task is to determine whether they should switch from the intuitive option to a non-intuitive alternative. In order to make this stay-switch decision, people consider two kinds of information. For one thing, people consider constraint information, a term that includes any available information that constrains the intuition's validity, that opposes the intuition, or that supports a non-intuitive alternative. For example, a manager's positive intuition about an interviewee is constrained by any negative information about the interviewee that the manager has available (e.g., knowledge that the candidate has a hankering for mid-afternoon naps). As with many models of judgment, we predict that strong constraint information will cause people to switch away from their intuitions more often than weak constraint information; this prediction hinges on whether the magnitude of the constraint information can be and is evaluated (Hsee, 2000). If people cannot properly evaluate the magnitude of the constraint information, then its magnitude should have little or no effect on choice. Although this hypothesis seems remarkably obvious, we will demonstrate that this effect occurs even when constraint magnitude has no informative value.

The second piece of information that people consider (and the piece most critical to this theory) is intuitive confidence. Generating an intuition often feels easy, but sometimes it can feel quite difficult. The more easily intuitions are generated, the more confidently people hold them. Thus, easily generated intuitions are held with high confidence, and those generated with difficulty are held with low confidence (e.g., Epley and Norwick, 2006). In line with a large body of research demonstrating that people rely on meta-cognitive feelings as information when they make decisions (e.g., Nelson and Morrison, 2005), it is suggested that people use intuitive confidence to decide whether to cling to an intuition when confronted with constraint information. High intuitive confidence signals 
intuitive accuracy and that the intuitive option should be chosen. In contrast, low intuitive confidence signals intuitive inaccuracy and that the non-intuitive option should perhaps be chosen. As a result, people will choose intuitive options with greater frequency as intuitive confidence increases.

Although intuitive confidence varies across decision contexts, it is also true that people typically have great confidence in their intuitions. Because intuitions spring to mind quickly, intuitions often feel easily generated, and this feeling increases intuitive confidence. On this basis, this research suggests two additional hypotheses. First, because people are often confident in their intuitions, even invalidated intuitions will be chosen more often than nonintuitive alternatives. Unlike existing theories, then, our theory assumes that the ubiquity of intuitive biases derives from the ubiquity of intuitive confidence, so that removing confidence will remove the bias. Second, because intuitions are more easily considered and more confidently held than non-intuitive alternatives, it is suggested that people who do choose non-intuitive alternatives will feel less confident in their choices. Thus, because intuitions feel so compelling, people will either choose them or find themselves stuck with an option that feels less compelling. In summary, four general predictions are made about how people choose between intuitive and non-intuitive alternatives. The first three hypotheses focus on aspects of making the decision, and the fourth focuses on consequences of the decision: 1. Intuitive Bias Hypothesis: Because intuitions are often held with high confidence, people will choose intuitive options more frequently than equally valid non-intuitive options, 2 . Constraint Magnitude Hypothesis: People will choose intuitive options less frequently when constraint information seems to more strongly favor a non-intuitive option; 3. Intuitive Confidence Hypothesis: People will choose intuitive options more frequently when they are more confident in their intuitions; 4. Intuitive Betrayal Hypothesis: People who betray their intuitions will feel less confident in their choices than people who choose in line with their intuitions.

In the research described subsequently, these four hypotheses were tested by investigating how people make predictions of sporting events. This choice domain offered many advantages. First, as described below, these predictions have all of the critical features of choice tasks to which we intend to generalize, including an intuitive option and an important constraint on the intuition's validity. Second, these choices present decision makers with naturally varying constraint magnitude and intuitive confidence, allowing the testing of the hypotheses in richly naturalistic settings as well as strictly controlled laboratory settings. Third, this domain presents decision makers with constraint information that is easy to understand and to process, allowing us to investigate intuitive choosing when it is clear that the constraint information has been processed. Fourth, decision makers in this domain often have money or pride at stake, enabling us to investigate how people make predictions when they are motivated to conduct extensive mental processing and to be accurate. Finally, these decisions have objective win-loss outcomes, allowing the assessment of the accuracy of people's decisions.

\section{MAKING PREDICTIONS AGAINST POINT SPREADS}

Imagine that a perennially dominant team (the New England Patriots) is about to play a weaker team (the Detroit Lions, more recently) in a National Football League (NFL) contest and that you want to wager some money on the game. Your bookmaker informs you that the Patriots are favored by a point spread of 14 points. This means that a bet on the Patriots pays out if the Patriots win by more than 14 points, whereas a bet on the Lions pays out if the Patriots win by less than 14 points or if the Lions win the game. (If the Patriots win by exactly 14 points, no bet wins, and no money changes hands.) Sophisticated gamblers know that point spreads are designed to be accurate (i.e., that they in fact represent the median of all possible game outcomes), but gamblers are nevertheless determined to make a wager. Or course, only a very sophisticated gambler knows this, it turns out. Readers familiar with gambling against point spreads may be surprised to learn that, contrary to popular belief, point spreads do not equate the amount of money bet on favorites and underdogs (Roxborough and Rhoden, 1998). Rather, point spreads are determined by casinos in Las Vegas, who rely on expert firms to set spreads that are designed to be accurate. (These firms also decide which team will serve as the favorite.) In fact, point spreads are remarkably effective (though not perfect) at equaling the probability of a favorite versus an underdog winning against the spread but, as we show, hopelessly far from equating the money bet on the favorite and underdog. Faced with the choice between two equally likely alternatives, you have to decide which one to choose. Which team would you put your money on? 
This scenario captures a decision that tens of thousands of people make each week when they gamble on sporting events. These gambles require a prediction between two alternatives that are equated by a point spread, which adds points to the expected losing team (the underdog) to even the probability of a winning bet on the underdog and the expected winner (the favorite). A bet on the favorite pays out if the favorite wins the game by more than the point spread. A bet on the underdog pays out if the favorite wins by less than the point spread or if the underdog wins the game.

How do people make these predictions? Consistent with Kahneman and Frederick (2002) and with the decision-making account that we developed above, we assume that people begin to answer such a difficult question (i.e., "Which team will win against the point spread?") by first quickly answering an easier one instead ("Which team will win the game?"). Because the favorite is often the better team (that is why it is favored, after all), people are often quick to identify it as such, and the favorite serves as the intuitive option. With this as a starting point, people subsequently consider the point spread (the constraint information). Specifically, they consider whether they should stay with the favorite or switch to choosing the underdog in light of the point spread's magnitude.

Because it is often very easy for people to determine that the favorite is superior, people will often be quite confident in this initial, intuitive assessment. This strong feeling of intuitive confidence will convince people to stay with their intuitions in the face of constraint information, causing them to predict favorites more frequently than underdogs against point spreads. Nevertheless, although the tendency to predict favorites should be common, it should also be predictably non-universal. When constraint magnitude is low (i.e., a low point spread) or intuitive confidence is high (i.e., when it is easy to identify the favorite as the superior team), people will tend to predict favorites. But when the point spread is high and/or intuitive confidence is low, people's predictions should be much more balanced: Large point spreads and uncertain intuitions will result in relatively more underdog predictions than low point spreads and confident intuitions.

In sum, in terms of the four general hypotheses proposed previously, we can expect the following about predictions of sporting events against point spreads: 1. Intuitive Bias Hypothesis: The favorite will often serve as a confidently held intuitive option, and people will predict favorites more frequently than they predict under dogs against the point spread; 2. Constraint Magnitude Hypothesis: People will predict favorites less frequently as point spread magnitude increases; 3. Intuitive Confidence Hypothesis: People will predict favorites less frequently as confidence in the favorite's relative superiority decreases; 4. Intuitive Betrayal Hypothesis: People who predict favorites against the spread will have greater confidence in this prediction than people who predict underdogs against the spread.

These specific hypotheses are interesting not only because of what they generally imply about how people make decisions in the face of invalid intuitions, but also because they are at odds with what one might expect from accuracy-motivated decision makers in this context. Because bets on favorites and underdogs are about equally likely to pay out (with underdogs winning slightly, but significantly, more often; Golec and Tamarkin, 1991; and Gray and Gray 1997), then, normatively speaking, people should be either equally likely to bet on favorites and underdogs or even more likely to bet on underdogs than favorites. Moreover, they should also have either equal confidence in their predictions of underdogs and favorites, or they should be more confident, overall, in underdog predictions. In addition, because the official point spread essentially equates the two teams, point spread magnitude and intuitive confidence are irrelevant with respect to accuracy. The probability of either team winning against the spread is the same no matter the point spread or one's level of intuitive confidence.

\section{A NATIONAL ON-LINE SAMPLE STUDY}

Every week of the football season, thousands of people log onto Yahoo.com's fantasy sports Web site http://fantasysports.yahoo.com to register their predictions about upcoming NFL and college (National Collegiate Athletic Association [NCAA]) football games. Some fantasy leagues require players to predict game outcomes against the point spread, whereas others require only the prediction of the game's winner. For each of these 
leagues, Yahoo.com publishes the percentage of people who predicted each team for each game, thereby providing us with a wealth of game-level data that enabled us to test the first three hypotheses described previously.

\section{METHOD}

For each game listed on the Yahoo.com Web site during the 2003 and 2004 NFL and NCAA football regular seasons, this research recorded (a) the point spread, (b) the percentage of people who predicted that the favorite would win against the spread, and (c) the percentage of people who predicted that the favorite would simply win the game. This last measure was used as an indication of game-level intuitive confidence, reasoning, for example, that a game featuring a favorite that is expected to win by $95 \%$ of the sample is associated with greater intuitive confidence than a game that features a favorite that is expected to win by $85 \%$ of the sample.

Games were eliminated from the analyses if the majority of people (i.e., more than 50\%) predicted that the underdog would simply win the game $(2003 \mathrm{NCAA}=6.8 \% ; 2004 \mathrm{NCAA}=6.3 \% ; 2003 \mathrm{NFL}=13.3 \%$; $2004 \mathrm{NFL}=15.2 \%$ ) because in these cases the point spread bolstered rather than constrained the majority's intuition. Also eliminated were games when the spread was zero or off the board (2003 NCAA $=0.4 \% ; 2004$ $\mathrm{NCAA}=1.8 \% ; 2003 \mathrm{NFL}=4.3 \% ; 2004 \mathrm{NFL}=4.3 \%$ ) because there is no favorite in these games; it is routine for casinos to refuse to take bets on (and set spreads for) upcoming games when there is uncertainty about a major aspect of the game (e.g., whether a mildly injured star player will be able to play). In total, predictions of 850 games were analyzed.

\section{RESULTS}

According to the intuitive bias hypothesis, people should predict favorites more often than underdogs against the point spread. Consistent with this hypothesis, Appendix reveals that the average percentage of people predicting favorites exceeded the chance expectation of 50\% in all four samples, averaging $70 \%$ across all 850 games $\left(\mathrm{t}>20, \mathrm{p}<10^{-52}\right)$. The appendix also displays the percentage of games in each sample that featured the majority of people predicting the favorite to win against the spread. Consistent with our hypothesis, favorites were chosen by the majority in more than $90 \%$ of the games in each of the four samples.

Predicting more favorites was not a winning strategy. Across all four samples of games, the favorite won against the spread $49.9 \%$ of the time (in 413 out of 828 games; there were 22 ties). This level of (in)effectiveness was not significantly different from $50 \%, \chi^{2}(1, \mathrm{~N}=828)=0.004, \mathrm{p}=.94$, and the tendency to predict favorites against the spread was wholly unrelated to any tendency for the favorite to actually beat the spread, $\mathrm{R}(826)=-$ $0.0002, \mathrm{p}=0.99$. The appendix shows that the tendency to predict favorites was equally strong in samples in which the favorite lost the majority of the time as in samples in which the favorite won the majority of the time.

This research is not the first to discover that people predict favorites more than underdogs against point spreads. Levitt (2004), in his economic analysis of bookmaking markets, observed a similar betting asymmetry with a smaller group of gamblers. As he pointed out, this finding is at odds not only with conventional wisdom but also with a seemingly rational description of bookmaking markets. He further argued that the asymmetry may offer profit opportunities for the craftiest bookmakers. But perhaps the most notable feature of Levitt's argument is what it misses rather than what it finds: It does not offer any explanation for why people are so predisposed toward favorites, opting instead for merely observing that people "exhibit a systematic bias toward favorites" (Levitt, 2004, p. 226). This psychological account and data allow the body of knowledge to move past the behavioral agnosticism of the economic approach. Simmons and Nelson (2006) call for an analyses peer within the black box of the "bias toward favorites" to learn where this and similar biases come from.

Thus, two additional predictions are made to test in this study. First, in line with the constraint magnitude hypothesis, it is predicted that fewer people would choose favorites as point spreads increased. Second, the intuitive confidence hypothesis predicted that increased consensus about whether the favorite would win the game (the game-level measure of intuitive confidence) would be associated with an increased tendency to 
predict favorites against the point spread. To test these hypotheses, this research regressed the percentage of people who predicted the favorite to win against the spread on (a) the point spread and (b) intuitive confidence. The results are displayed in the right-most columns of the appendix. In support of the constraint magnitude hypothesis, people were less likely to predict the favorite if the game featured a larger point spread. In support of the intuitive confidence hypothesis, people were more likely to predict the favorite against the spread if they were more likely to think the favorite would simply win the game.

Because intuitive confidence and point spread magnitude are positively correlated (i.e., high confidence games tend to feature higher point spreads; $\mathrm{R}$ ranging from 0.59 to 0.74 across the four Yahoo.com samples) and because it is expected that the effects of intuitive confidence and point spread magnitude to run in opposite directions (i.e., high constraint magnitude $=$ fewer favorite predictions; high confidence $=$ more favorite predictions), these variables were entered simultaneously in the above regression to reveal their unique effects. However, to assess the robustness of these effects, also analyzed were the separate, non-unique effects of each of these variables as well. When analyzed separately, point spread magnitude exerted no consistent effect on against-the-spread predictions, with Rs ranging from negative 0.15 to 0.16 across the four samples. However, attesting to the robustness of the effect, intuitive confidence remained a highly significant predictor of against-thespread predictions even in the absence of point spread magnitude, with Rs ranging from 0.54 to 0.69 (all $\mathrm{p}<10^{-16}$ ) across the four samples. This indicates that, no matter the magnitude of the point spread, people predicted favorites against the spread more often as intuitive confidence increased.

The effects of constraint magnitude and intuitive confidence arose despite the fact that these variables were unrelated to prediction accuracy. Neither point spread magnitude nor intuitive confidence correlated with whether the favorite beat the spread, whether these analyses controlled for the other variable or not (all Rs between -0.025 and $0.015 ; \mathrm{p}>.51$ ).

\section{DISCUSSION}

This study provides very strong evidence that people predict favorites more often than underdogs against the point spread. The majority predicted the favorite against the spread in more than $90 \%$ of the games, though the favorite won slightly less than $50 \%$ of the time. The results of this study strongly supported the intuitive confidence and constraint magnitude hypotheses as well. The percentage of people predicting favorites against the spread was positively related to the percentage who believed that the favorite would simply win the game and negatively related to the size of the point spread.

\section{CONCLUSION}

This article has developed an account that explains why intuitions frequently pervade the choices that people make. Specifically, it has been proposed that intuitive biases arise in large part because of intuitive confidence and that decreasing confidence in the intuition will decrease, or even eliminate, such biases. In so doing, it is uniquely predicted when intuitive biases will and will not occur, while offering a promising plan for reducing them. 


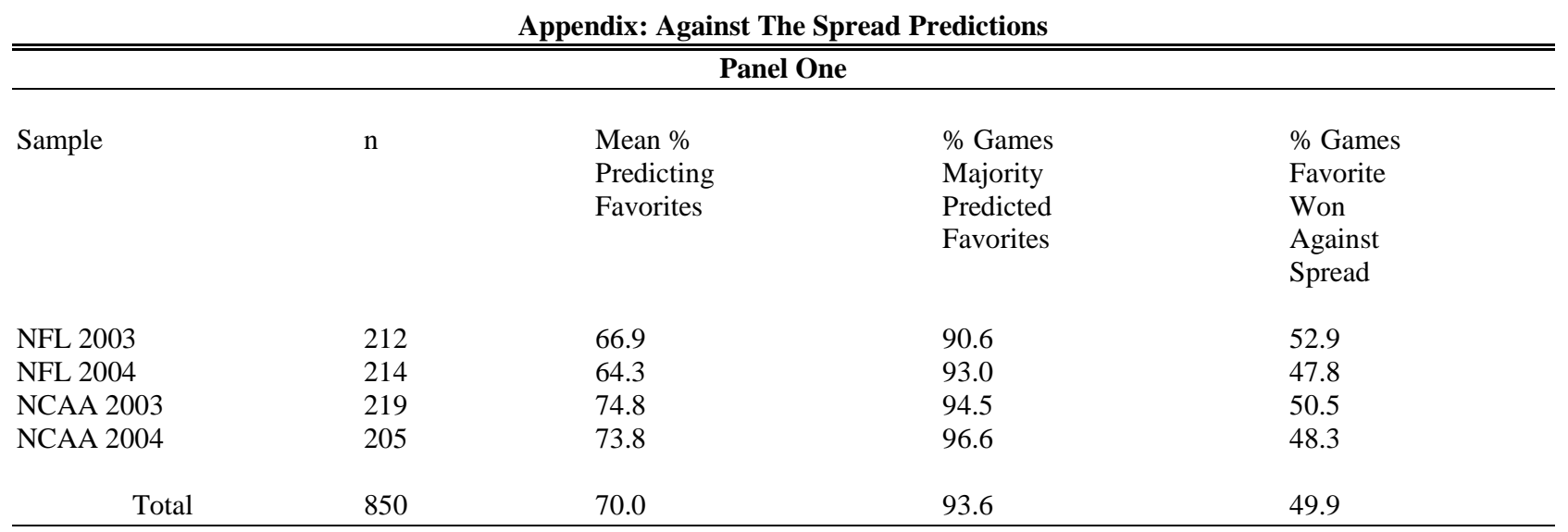

\begin{tabular}{|c|c|c|c|c|c|c|}
\hline \multicolumn{7}{|c|}{ Panel Two } \\
\hline & \multicolumn{3}{|c|}{$\begin{array}{l}\text { Effect of Point } \\
\text { Spread Magnitude }\end{array}$} & \multicolumn{3}{|c|}{$\begin{array}{l}\text { Effect of } \\
\text { Intuitive Confidence }\end{array}$} \\
\hline & $\beta$ & SE & $\mathrm{p}<$ & $\beta$ & SE & $\mathrm{p}<$ \\
\hline NFL 2003 & -3.47 & 0.17 & $10-52$ & 1.01 & 0.04 & $10-73$ \\
\hline NFL 2004 & -3.30 & 0.19 & $10-42$ & 0.98 & 0.04 & $10-58$ \\
\hline NCAA 2003 & -0.71 & 0.07 & $10-18$ & 1.09 & 0.06 & $10-47$ \\
\hline NCAA 2004 & -0.69 & 0.08 & $10-15$ & 1.05 & 0.07 & $10-32$ \\
\hline Total & -0.41 & 0.05 & $10-18$ & 0.81 & 0.03 & $10-112$ \\
\hline
\end{tabular}

\section{REFERENCES}

1. Cacioppo, J. and R. Petty, 1982. The Need for Cognition, Journal of Personality and Social Psychology, pp. $116-131$.

2. Chaiken, S., A. Liberman, and A. Eagly, 1989. Heuristic and Systematic Processing Within and Beyond the Persuasion Context, In J. Uleman and J. Bargh (Eds.), Unintended Thought (pp. 212-252). New York: Guilford Press.

3. Cheri, S. and S. Chaiken, S., 1999. The Heuristic-Systematic Model in Its Broader Context, In S. Chaiken and Y. Trope (Eds.), Dual-Process Theories in Social Psychology (pp. 73-96). New York: Guilford Press.

4. $\quad$ Dawes, R., D. Faust, and P. Meehl, 1989. Clinical versus Actuarial Judgment, Science, pp. 1668-1674.

5. Denes-Raj, V. and S. Epstein, 1994. Conflict between Intuitive and Rational Processing: When People Behave against Their Better Judgment, Journal of Personality and Social Psychology, pp. 819-829.

6. Denes-Raj, V., S. Epstein, S., and J. Cole, 1995. The Generality of the Ratio-Bias Phenomenon, Personality and Social Psychology Bulletin, pp. 1083-1092.

7. Epley, N. and T. Gilovich, 2004. Are Adjustments Insufficient? Personality and Social Psychology Bulletin, pp. 447-460.

8. $\quad$ Epley, N. and T. Gilovich, 2006. The Anchoring-and-Adjustment Heuristic, Psychological Science, pp. 311318.

9. Epley, N., B. Keysar, B., L. Van Boven, and T. Gilovich, 2004. Perspective Taking as Egocentric Anchoring and Adjustment, Journal of Personality and Social Psychology, pp. 327-339.

10. Epley, N. and R. Norwick, 2006. Confidence as Inference from Incidental Experience, Unpublished Manuscript, University of Chicago. 
11. Epstein, S., 1994. Integration of the Cognitive and the Psychodynamic Unconscious, American Psychologist, pp. $709-724$.

12. Fischhoff, B., 1975. Hindsight is not Equal to Foresight: The Effect of Outcome Knowledge on Judgment under Uncertainty, Journal of Experimental Psychology: Human Perception and Performance, pp. 288-299.

13. Gilbert, D., 1991. How Mental Systems Believe, American Psychologist, pp. 107-119.

14. Gilbert, D., 2002. Inferential Correction, In T. Gilovich, D. Griffin, and D. Kahneman (Eds.), Heuristics and Biases: The Psychology of Intuitive Judgment (pp. 167-184). Cambridge, United Kingdom:

Cambridge University Press.

15. Gilbert, D., B. Pelham, and D. Krull, 1988. On Cognitive Business: When Person Perceivers Meet Persons Perceived, Journal of Personality and Social Psychology, pp. 733-740.

16. Gilovich, T., D. Griffin, D., and D. Kahneman, (Eds.), 2002. Heuristics and Biases: The Psychology of Intuitive Judgment. Cambridge, United Kingdom: Cambridge University Press.

17. Gilovich, T., V. Medvec, and K. Savitsky, 2000. The Spotlight Effect in Social Judgment: An Egocentric Bias in Estimates of the Salience of One's Own Actions and Appearance, Journal of Personality and Social Psychology, pp. 211-222.

18. Gilovich, T., K. Savitsky, and V. Medvec, 1998. The Illusion of Transparency: Biased Assessments of Others' Ability to Read Our Emotional States, Journal of Personality and Social Psychology, pp. 332-346.

19. Gladwell, M., 2004, January 12. Big and Bad: How the S.U.V. Ran Over Automotive Safety, The New Yorker, pp. 28-33.

20. Golec, J. and M. Tamarkin, 1991. The Degree of Inefficiency in the Football Betting Market, Journal of Financial Economics, pp. 311-323.

21. Gray, P. and S. Gray, 1997. Testing Market Efficiency: Evidence from the NFL Sports Betting Market, Journal of Finance, pp. 1725-1737.

22. Hsee, C., 2000. Attribute Evaluability: Its Implications for Joint-Separate Evaluation Reversals and Beyond, In D. Kahneman and A. Tversky (Eds.), Choices, Values, and Frames (pp. 543-564). Cambridge, United Kingdom: Cambridge University Press.

23. Jones, E., V. and Harris, 1967. The Attribution of Attitudes, Journal of Experimental Social Psychology, pp. 1-24.

24. Kahneman, D., 2003. A Perspective on Judgment and Choice: Mapping Bounded Rationality, American Psychologist, pp. 697-720.

25. Kahneman, D. and S. Frederick, S, 2002. Representativeness Revisited: Attribute Substitution in Intuitive Judgment, In T. Gilovich, D. Griffin, D. Kahneman (Eds.), Heuristics and Biases: The Psychology of Intuitive Judgment (pp. 49-81). Cambridge, United Kingdom: Cambridge University Press.

26. Kruger, J., 1999. Lake Wobegon be Gone! The Below-Average Effect and the Egocentric Nature of Comparative Ability Judgments, Journal of Personality and Social Psychology, pp. 221-232.

27. Levitt, S., 2004. Why are Gambling Markets Organized so Differently from Financial Markets? The Economic Journal, pp. 223-246.

28. Moore, D. and T. Kim, 2003. Myopic Social Prediction and the Solo Comparison Effect, Journal of Personality and Social Psychology, pp. 1121-1135.

29. Nelson, L. and E. Morrison, 2005. The Symptoms of Resource Scarcity: Judgments of Mood and Finances Influence Preferences for Potential Partners, Psychological Science, pp. 167-173.

30. Pelham. B. and E. Neter, 1995. The Effect of Motivation on Judgment Depends on the Difficulty of the Judgment, Journal of Personality and Social Psychology, pp. 581-594.

31. Petty, R. and D. Wegener, 1999. The Elaboration Likelihood Model: Current Status and Controversies, In S. Chaiken and Y. Trope (Eds.), Dual-Process Theories in Social Psychology (pp. 73-96). New York: Guilford Press.

32. Roxborough, R. and M. Rhoden, 1998. Sports Book Management: A Guide for the Legal Bookmaker. Las Vegas, NV: Las Vegas Sports Consultants.

33. Simonson, I. and A. Tversky, 1992. Choice in Context: Tradeoff Contrast and Extremeness Aversion, Journal of Marketing Research, pp. 281-295.

34. Simmons, J. and L. Nelson, 2006. Intuitive Confidence: Choosing Between Intuitive and Nonintuitive Alternatives, Journal of Experimental Psychology: General, pp. 409-428. 
35. Todorov, A., A. Mandisodza, A. Goren, and C. Hall, 2005. Inferences of Competence from Faces Predict Election Outcomes, Science, pp. 1623-1626.

\section{NOTES}

